\title{
On the computation and parametrization of proper denominator assigning compensators for strictly proper plants
}

\author{
E. N. Antoniou* A.I.G. Vardulakis \\ Department of Mathematics \\ Aristotle University of Thessaloniki \\ 54006 - Thessaloniki -GREECE \\ Tel: +30 2310997951 - Fax: +302310997951 \\ E-mail: antoniou@math.auth.gr, avardula@auth.gr
}

February 13, 2004

\begin{abstract}
Given a right coprime MFD of a strictly proper plant $P(s)=N_{R}(s) D_{R}(s)^{-1}$ with $D_{R}(s)$ column proper a simple numerical algorithm is derived for the computation of of all polynomial solutions $\left[X_{L}(s), Y_{L}(s)\right]$ of the polynomial matrix Diophantine equation $X_{L}(s) D_{R}(s)+Y_{L}(s) N_{R}(s)=D_{C}(s)$ which give rise to the class $\Phi\left(P, D_{C}\right)$ of proper compensators $C(s):=$ $X_{L}(s)^{-1} Y_{L}(s)$ that when employed in a unity feedback loop result to closed loop systems $S(P, C)$ with a desired denominator $D_{C}(s)$. The parametrization of the proper compensators $C(s) \in \Phi\left(P, D_{C}\right)$ is obtained and the number of independent parameters in the parametrization is given.
\end{abstract}

\section{Introduction}

We consider linear, time invariant, multivariable systems which are assumed to be free of unstable hidden modes and whose input-output relation is described by a strictly proper transfer function matrix $P(s)$ (the plant). In this note we describe a numerically efficient algorithm for the computation of the class of proper compensators $C(s)$ which, when employed in the unity feedback loop of figure 1, gives rise to a closed loop system $S(P, C)$ with a specific closed

${ }^{*}$ Corresponding author. Dr. Antoniou's research is supported by the Greek Scholarship Foundation (IKY) (Postdoctoral research grant, Contract Number 411/2003-04) 


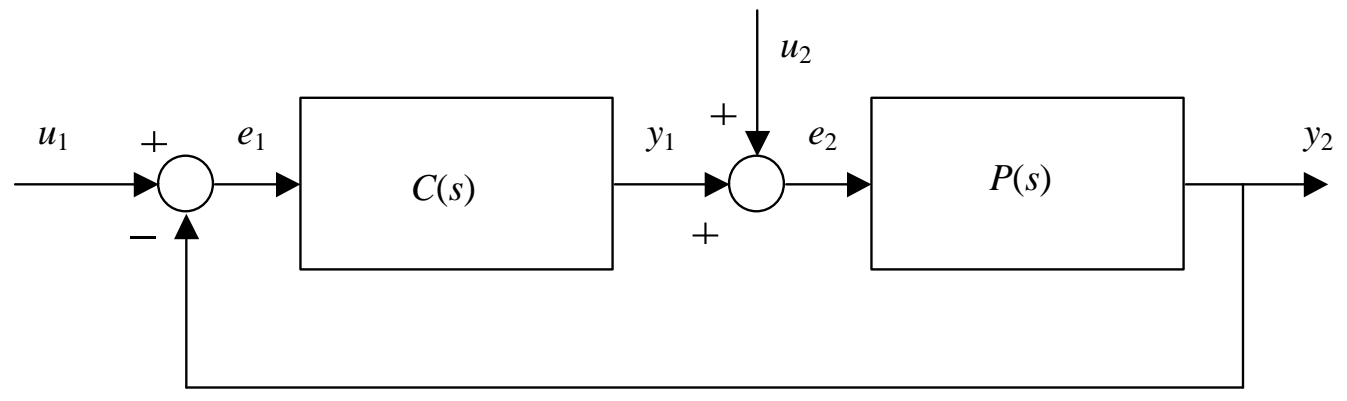

Figure 1: The unity Feedback system $S(P, C)$

loop denominator $D_{C}(s)$ [6], [8]. In particular, given a right coprime MFD of a strictly proper plant $P(s)=N_{R}(s) D_{R}(s)^{-1}$ with $D_{R}(s)$ column proper (column reduced) and an appropriately defined polynomial matrix $D_{C}(s)$ with desired zeros, we extend the Wolovich [1] resultant theorem and a theorem by Callier and Desoer [13], Callier [14] and Kucera [9] in order to obtain an algorithm for the computation of all polynomial solutions $\left[X_{L}(s), Y_{L}(s)\right]$ of the polynomial matrix Diophantine equation

$$
X_{L}(s) D_{R}(s)+Y_{L}(s) N_{R}(s)=D_{C}(s)
$$

which give rise to the class $\Phi\left(P, D_{C}\right)$ of proper compensators $C(s):=X_{L}(s)^{-1} Y_{L}(s)$ that result to closed loop systems $S(P, C)$ with $D_{C}(s)$ as their closed-loop denominator. The issues of the parametrization of the proper compensators $C(s) \in \Phi\left(P, D_{C}\right)$ and the number of independent parameters in the parametrization is also resolved. This is done by investigating the properties of a generalized version of Wolovich's resultant to obtain a series of new results regarding its algebraic structure. Despite the fact that similar results for Sylvester-type resultants have been presented in [3], the Wolovich resultant has not received the expected attention, except perhaps [1] and [2] where Wolovich's resultant is used as a tool for testing the coprimeness of polynomial matrices.

The method presented here can be compared to the one in [11], where Wolovich's resultant is employed as a tool for the construction of the interpolation matrix. However, our method requires only knowledge of the coefficients of the polynomial matrices $D_{R}(s), N_{R}(s)$ and provides a parametrization of all proper denominator assigning controllers unifying in this way the "resultant" approach with the approaches in [13], [14] and [9]. The proposed approach can be viewed as a generalization of the method presented in [10] (theorem 2.13 , p. 547) where the solution of a degree-specific Diophantine equation is obtained using Wolovich's resultant. Furthermore, through the investigation of the rank of the generalized Wolovich resultant, we establish the lower bound for the (McMillan) degree of an arbitrary closed loop denominator, a fact which has been used throughout the constructions in [13], [14], [9], but not justified 
via some theoretic argument.

\section{Preliminaries}

In the following $\mathbb{R}, \mathbb{C}, \mathbb{R}(s), \mathbb{R}[s], \mathbb{R}_{p r}(s), \mathbb{R}_{p o}(s)$ are respectively the fields of real numbers, complex numbers, real rational functions, the rings of polynomials, proper rational and strictly proper rational functions all with coefficients in $\mathbb{R}$ and indeterminate $s$. For a set $\mathbb{F}, \mathbb{F}^{p \times m}$ denotes the set of $p \times m$ matrices with entries in $\mathbb{F}$. $\mathbb{N}^{+}$is the set of positive integers. If $m \in \mathbb{N}^{+}$then $\mathbf{m}$ denotes the set $\{1,2, \ldots, m\}$. Finally $\delta_{M}[$.$] denotes the McMillan degree of [.]$

Let $N_{R}(s) \in \mathbb{R}[s]^{p \times m}, D_{R}(s) \in \mathbb{R}[s]^{m \times m}$ be a pair of polynomial matrices with $D_{R}(s)$ invertible for almost every $s \in \mathbb{C}$ and define the compound matrix $F(s):=\left[D_{R}^{\top}(s), N_{R}^{\top}(s)\right]^{\top}$. Respectively let $D_{L}(s) \in \mathbb{R}[s]^{p \times p}, N_{L}(s) \in \mathbb{R}[s]^{p \times m}$ (with $D_{L}(s)$ invertible for a.e. $s \in \mathbb{C}$ ) and $E(s):=\left[-N_{L}(s), D_{L}(s)\right]$ such that

$$
E(s) F(s)=0
$$

The pair of matrices $N_{R}(s), D_{R}(s)$ (resp. $N_{L}(s), D_{L}(s)$ ) will be called right (resp. left) coprime iff $F(s)$ has full column rank (resp. $E(s)$ has full row rank) for every $s \in \mathbb{C}$. It is known that $N_{R}(s), D_{R}(s)$ are right coprime and $N_{L}(s), D_{L}(s)$ left coprime, then

$$
\operatorname{deg}\left|D_{R}(s)\right|=\operatorname{deg}\left|D_{L}(s)\right|
$$

A polynomial matrix $X(s) \in \mathbb{R}[s]^{p \times m}(m \leq p)$ is called column proper or column reduced iff its highest column coefficient matrix denoted $X^{h c}$ which is formed by the coefficients of the highest degrees of $s$ in each column of $X(s)$, has full column rank. The column degrees of $X(s)$ are usually denoted by $\operatorname{deg}_{c i} X(s)$, $i \in \mathbf{m}$. Respectively $Y(s) \in \mathbb{R}[s]^{p \times m}(p \leq m)$ is called row proper or row reduced iff $Y^{T}(s)$ is column proper and the row degrees of $Y(s)$ are denoted by $\operatorname{deg}_{r i} Y(s), i \in \mathbf{p}$. Furthermore a square polynomial matrix $X(s) \in \mathbb{R}[s]^{m \times m}$ is called row-column reduced [13] with row powers $r_{i}$ and column powers $c_{i}, i \in \mathbf{m}$ iff the matrix $\underset{i \in \mathbf{m}}{\operatorname{diag}}\left\{s^{-r_{i}}\right\} X(s) \underset{i \in \mathbf{m}}{\operatorname{diag}}\left\{s^{-c_{i}}\right\}$ is biproper (i.e. it is proper and its inverse exists and it is proper as well).

Lemma 1 [5] (Corollary 3.100, p. 144) If $X(s) \in \mathbb{R}[s]^{p \times m}(p \geq m)$ is column proper then $X(s)$ has no zeros at infinity and its (ordered) column degrees are the orders of its poles at infinity i.e. if

$$
S_{X(s)}^{\infty}=\left[\begin{array}{l}
\operatorname{diag}\left\{s^{q_{1}}, s^{q_{2}}, \ldots, s^{q_{m}}\right\} \\
0_{p-m, m}
\end{array}\right]
$$

is the Smith - McMillan form of $X(s)$ at infinity, with $q_{1} \geq q_{2} \geq \ldots \geq q_{m} \geq 0$, then $q_{i}=\operatorname{deg}_{c i} X(s), i \in \mathbf{m}$. Furthermore since $X(s)$ (as polynomial matrix) has no finite poles and due to $s^{q_{i}}$ has (possibly) only poles at infinity, $\delta_{M} X(s)=$ $\sum_{i=1}^{m} \operatorname{deg}_{c i} X(s)$. 
Obviously a similar result holds for row proper matrices..

When (2) is satisfied and $E(s)$ is row proper with $D_{L}(s), N_{L}(s)$ left coprime, $E(s)$ is a minimal polynomial basis of the (rational) vector space spanning the left kernel of $F(s)$ and the row degrees $\operatorname{deg}_{r i} E(s)=: \mu_{i}, i \in \mathbf{p}$ of $E(s)$ are the invariant row minimal (dual) dynamical indices of

$$
P(s)=N_{R}(s) D_{R}^{-1}(s)=D_{L}^{-1}(s) N_{L}(s)
$$

In such a case it is known [4] that $E(s)$ has the following properties

1. If $p(s)^{\top} \in \mathbb{R}[s]^{1 \times(p+m)}$ is a polynomial vector such that $p(s)^{\top} F(s)=0$ then there exists a polynomial vector $w(s)^{\top}=\left[w_{1}(s), w_{2}(s), \ldots, w_{p}(s)\right] \in$ $\mathbb{R}[s]^{1 \times p}$ such that

$$
p(s)^{\top}=w(s)^{\top} E(s)
$$

2. If $p(s)^{\top}=w(s)^{\top} E(s)$ then

$$
\operatorname{deg} p(s)^{\top}=\max _{i \in \mathbf{p}}\left\{\operatorname{deg} w_{i}(s)+\mu_{i}\right\}
$$

The following result establishes a relation between the McMillan degrees of $P(s)$ and $E(s)$ (or $F(s))$.

Lemma $2[5]$ (p. 140) If $E(s)$ has no zeros in $\mathbb{C} \cup\{\infty\}$ (equiv. $D_{L}(s), N_{L}(s)$ are left coprime in $\mathbb{C} \cup\{\infty\})$ then

$$
\delta_{M} P(s)=\delta_{M} E(s)
$$

When $E(s)$ is a minimal polynomial basis of the left kernel of $F(s)$, i.e. $E(s)$ has no zeros in $\mathbb{C}$ and is row proper, by lemma $1 E(s)$ will have no zeros in $\mathbb{C} \cup\{\infty\}$ and thus from the last statement of lemma 1

$$
\delta_{M} P(s)=\delta_{M} E(s)=\sum_{i=1}^{p} \operatorname{deg}_{r i} E(s)
$$

Furthermore if also $D_{R}(s), N_{R}(s)$ are right coprime and $F(s)$ is column proper then again from lemma 1 and lemma 2

$$
\delta_{M} P(s)=\delta_{M} F(s)=\sum_{i=1}^{m} \operatorname{deg}_{c i} F(s)
$$

thus in such a case we get the well known result [4] that

$$
\sum_{i=1}^{p} \operatorname{deg}_{r i} E(s)=\sum_{i=1}^{m} \operatorname{deg}_{c i} F(s)
$$




\section{Generalized Wolovich Resultant}

Let $k_{i}=\operatorname{deg}_{c i} F(s), i \in \mathbf{m}$ be the invariant minimal column dynamical indices of $F(s)$ and similarly to [1] (page 242) for $k \geq 1$ define the $(m+p) k \times m$ polynomial matrix $X_{k}(s)$ via

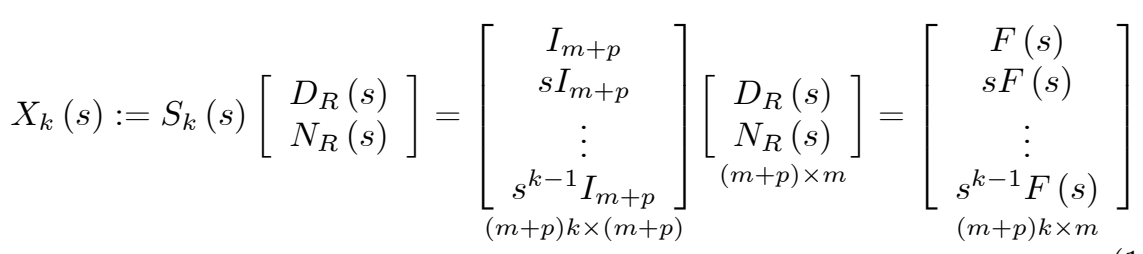

and notice that $X_{k}(s)$ can be written

$$
\left.\left.X_{k}(s)=M_{e k}\left[\begin{array}{c}
\text { block diag } \\
i \in \mathbf{m} \\
\left(\sum_{i=1}^{m} k_{i}+m k\right) \times m \\
s^{k_{i}+k-1}
\end{array}\right]\right\}\right]=: M_{e k} S_{e k}(s)
$$

where $M_{e k} \in \mathbb{R}^{(m+p) k \times\left(m k+\sum_{i=1}^{m} k_{i}\right)}$. Notice that $M_{e k}$ does not coincide with the one in [1] since Wolovich assumes that $D_{R}(s)$ is column proper and $P(s)=$ $N_{R}(s) D_{R}^{-1}(s)$ is proper. Apart of that essentially the two matrices differ only up to row permutations.

One of our goals is to describe the left null space (kernel) of $M_{e k}$ which in what follows is denoted

$$
\operatorname{Ker} M_{e k}^{\top}=\left\{x^{\top} \in \mathbb{R}^{1 \times(m+p) k}: x^{\top} M_{e k}=0\right\}
$$

The following theorem determines the dimension of $\operatorname{Ker} M_{e k}^{\top}$.

Theorem 3 Let $N_{R}(s) \in \mathbb{R}[s]^{p \times m}, D_{R}(s) \in \mathbb{R}[s]^{m \times m}$ be a pair of polynomial matrices with rank $\mathbb{R}(s)_{\mathbb{R}}\left[D_{R}^{\top}(s), N_{R}^{\top}(s)\right]^{\top}=m$. Let also $P(s)=N_{R}(s) D_{R}^{-1}(s) \in$ $\mathbb{R}(s)^{p \times m}, \mu_{i}, i \in \mathbf{p}$ be the invariant row minimal dynamical indices of $P(s)$ and $M_{e k} \in \mathbb{R}^{(m+p) k \times\left(m k+\sum_{i=1}^{m} k_{i}\right)}$ defined in (12). Then

$$
\operatorname{dim}_{\mathbb{R}} \operatorname{ker} M_{e k}^{\top}=\sum_{i: \mu_{i} \leq k}\left(k-\mu_{i}\right)
$$

Proof. The proof is identical to that of Theorem 1 in [3].

It is interesting to notice that the dimension of the kernel obtained here is identical to the one given in theorem 1 in [3], despite the fact that the generalized Sylvester resultant $S_{k}$ in [3] does not coincide in general with $M_{e k}$. Notice also that the above result does not require $D_{R}(s), N_{R}(s)$ to be right coprime nor 
$D_{R}(s)$ to be column proper. We give now a generalization of the result that appears in [11] (Lemma 3.2), in the sense that we relax $P(s)=N_{R}(s) D_{R}^{-1}(s)$ from the properness requirement as well as from the assumption that $D_{R}(s)$ is column proper.

Corollary 4 Under the assumptions of theorem 3, we have

$$
\operatorname{rank} M_{e k}=(p+m) k-\sum_{i: \mu_{i} \leq k}\left(k-\mu_{i}\right)
$$

Furthermore if $k$ is chosen s.t. $k \geq \mu$, where $\mu=\max _{i \in \mathbf{p}}\left\{\mu_{i}\right\}$ then

$$
\operatorname{rank} M_{e k}=m k+\delta_{M} P(s)
$$

Proof. Equation (15) follows simply from the fact that $r a n k M_{e k}=(p+m) k-$ $\operatorname{dim}_{\mathbb{R}} \operatorname{ker} M_{e k}^{\top}$ and equation (14). Now for $k \geq \mu$ (15) becomes $\operatorname{rank} M_{e k}=$ $(p+m) k-\sum_{i=1}^{p}\left(k-\mu_{i}\right)$ or equivalently $\operatorname{rank} M_{e k}=m k+\sum_{i=1}^{p} \mu_{i}$ thus (16) follows from the facts $\sum_{i=1}^{p} \mu_{i}=\delta_{M} E(s)=\delta_{M} P(s)$ in lemmata 1 and 2 .

Notice that in case $D_{R}(s)$ is column proper and $P(s):=N_{R}(s) D_{R}^{-1}(s)$ is proper, $\delta_{M} P(s)=\{\#$ of poles of $P(s)$ in $\mathbb{C}\}=\operatorname{deg}\left|D_{R}(s)\right|$. Therefore, for $k \geq \mu$ the above result coincides with the result of Lemma 3.2 in [11]. The following corollary provides a generalization of the corresponding result in [1] (page 242).

Corollary 5 Let $N_{R}(s) \in \mathbb{R}[s]^{p \times m}, D_{R}(s) \in \mathbb{R}[s]^{m \times m}$ be a pair of polynomial matrices with $F(s)=\left[D_{R}^{\top}(s), N_{R}^{\top}(s)\right]^{\top}$ column proper with column degrees $\operatorname{deg}_{c i} F(s)=k_{i}, i \in \mathbf{m}$. Then $N_{R}(s), D_{R}(s)$ are right coprime in $\mathbb{C}$ iff $M_{e k}$ has full column rank for $k \geq \mu$, or equivalently if $F(s)=\left[D_{R}^{\top}(s), N_{R}^{\top}(s)\right]^{\top}$ is column proper then $N_{R}(s), D_{R}(s)$ are right corpime in $\mathbb{C}$ iff for $k \geq \mu$, $\operatorname{rank} M_{e k}=m k+\delta_{M} F(s)$.

Proof. First notice that from (12) the number of columns in $M_{e k}$ is $m k+$ $\sum_{i=1}^{m} k_{i}$. Since $F(s)$ is column proper it has no zeros at infinity and from lemma $1 \sum_{i=1}^{m} k_{i}=\delta_{M} F(s)$. Hence the number of columns in $M_{e k}$ is $m k+\delta_{M} F(s)$.

$(\Rightarrow)$ Let $N_{R}(s), D_{R}(s)$ be right coprime in $\mathbb{C}$. Then from Corollary 4 for $k \geq \mu, \operatorname{rank} M_{e k}=k m+\delta_{M} P(s)$. Since $F(s)$ is column proper from lemma 1 it has no zeros at infinity, thus $N_{R}(s), D_{R}(s)$ are right coprime at $s=\infty$. Hence $N_{R}(s), D_{R}(s)$ are right coprime in $\mathbb{C} \cup\{\infty\}$, thus from lemma $2 \delta_{M} P(s)=$ $\delta_{M} F(s)$ and $\operatorname{rank}_{e k}=k m+\delta_{M} F(s)$.

$(\Leftarrow)$ Assume that $N_{R}(s), D_{R}(s)$ are not right coprime in $\mathbb{C}$. Then there exists $0 \neq x \in \mathbb{R}^{m \times 1}$ and $s_{0} \in \mathbb{C}$ such that $F\left(s_{0}\right) x=0$. In view of (12)

$$
X_{k}\left(s_{0}\right) x=M_{e k} S_{e k}\left(s_{0}\right) x=0
$$

hence $M_{e k}$ does not have full column rank.

The following remark establishes the fact that $M_{e k}$ can have full column rank only for $k \geq \mu$. 
Remark 6 Let $D_{R}(s) \in \mathbb{R}[s]^{m \times m}, N_{R}(s) \in \mathbb{R}[s]^{m \times m}$ such that $D_{R}(s), N_{R}(s)$ be right coprime in $\mathbb{C}$ and $F(s)=\left[D_{R}^{\top}(s), N_{R}^{\top}(s)\right]^{\top}$ be column proper with column degrees $\operatorname{deg}_{c i} F(s)=k_{i}, i \in \mathbf{m}$. Let also $\mu_{i}, i \in \mathbf{p}$ be the left minimal indices of $F(s)$ and define $\mu=\max _{i \in \mathbf{p}}\left\{\mu_{i}\right\}$. Then for $k<\mu$

$$
\operatorname{rank} M_{e k}<m k+\sum_{i=1}^{m} k_{i}
$$

i.e. $M_{e k}$ cannot have full column rank for $k<\mu$.

Proof. Assume $k<\mu$ and let $a$ be the number of $\mu_{i}$ 's satisfying $\mu_{i}>k$. It is easy to see that

$$
k a<\sum_{i: \mu_{i}>k} \mu_{i}
$$

Using the fact that $\sum_{i=1}^{p} \mu_{i}=\sum_{i: \mu_{i}>k} \mu_{i}+\sum_{i: \mu_{i} \leq k} \mu_{i}$ we can write (18) as $k a+$ $\sum_{i: \mu_{i} \leq k} \mu_{i}<\sum_{i=1}^{p} \mu_{i}$ or equivalently $\mathrm{AS}$

$$
p k-k(p-a)+\sum_{i: \mu_{i} \leq k} \mu_{i}<\sum_{i=1}^{p} \mu_{i}
$$

Notice that the number of terms in $\sum_{i: \mu_{i} \leq k} \mu_{i}$ is exactly $p-a$, thus we can write (19) as

$$
p k-\sum_{i: \mu_{i} \leq k}\left(k-\mu_{i}\right)<\sum_{i=1}^{p} \mu_{i}
$$

Adding $m k$ on both sides of $(20)$ we get $(m+p) k-\sum_{i: \mu_{i} \leq k}\left(k-\mu_{i}\right)<m k+$ $\sum_{i=1}^{p} \mu_{i}$ where obviously the left hand side is $\operatorname{rank} M_{e k}$ and $\sum_{i=1}^{p} \mu_{i}=\sum_{i=1}^{m} k_{i}$ due to the assumptions of coprimeness (of $D_{R}(s), N_{R}(s)$ in $\mathbb{C}$ ) and the column properness of $F(s)$ (see 10). Thus (17) follows.

The above result has a direct implication on the choice of the row degrees of $D_{C}(s)$ in equation (1) which will be discussed in the following section

\section{Application to matrix Diophantine equations}

Consider a strictly proper linear multivariable plant, $P(s) \in \mathbb{R}_{p o}(s)^{p \times m}$ with $m$ inputs and $p$ outputs and let

$$
P(s)=N_{R}(s) D_{R}(s)^{-1}=D_{L}(s)^{-1} N_{L}(s)
$$

be respectively right and left coprime MFDs of $P(s)$ with $N_{R}(s) \in \mathbb{R}[s]^{p \times m}$ and $D_{R}(s) \in \mathbb{R}[s]^{m \times m}$ and column proper with column degrees $\operatorname{deg} D_{R c i}(s)=$ 
$k_{i}, i \in \mathbf{m}, N_{L}(s) \in \mathbb{R}[s]^{p \times m}$ and $D_{L}(s) \in \mathbb{R}[s]^{p \times p}$ and row proper with row degrees $\operatorname{deg} D_{L r i}(s)=\mu_{i}, i \in \mathbf{p}$. Define $\mu:=\max _{i \in \mathbf{p}}\left\{\mu_{i}\right\}$ (the observability index of $P(s))$.

The problem of assigning the denominator of the closed-loop system using unity feedback and a dynamic precompensator $C(s) \in \mathbb{R}(s)^{m \times p}$, can be reduced to the solution of the polynomial matrix Diophantine equation of the form

$$
X_{L}(s) D_{R}(s)+Y_{L}(s) N_{R}(s)=D_{C}(s)
$$

where $D_{C}(s) \in \mathbb{R}[s]^{m \times m}$ is the desired closed-loop denominator matrix and $X_{L}(s) \in \mathbb{R}[s]^{m \times m}, Y_{L}(s) \in \mathbb{R}[s]^{m \times p}$ is a left (not necessarily coprime) MFD of $C(s)$, i.e.

$$
C(s)=X_{L}(s)^{-1} Y_{L}(s) \in \mathbb{R}(s)^{m \times p}
$$

It is well known that $(22)$ has a solution for arbitrary $D_{C}(s)$ iff $D_{R}(s)$, $N_{R}(s)$ are right coprime. Furthermore if $\bar{X}_{L}(s), \bar{Y}_{L}(s)$ is a particular solution of (22) then every pair of the form $X_{L}(s)=\bar{X}_{L}(s)+T(s) N_{L}(s), Y_{L}(s)=$ $\bar{Y}_{L}(s)-T(s) D_{L}(s)$ is also a solution of $(22)$ for any arbitrary polynomial matrix $T(s) \in \mathbb{R}[s]^{m \times p}$.

However, the question usually posed is under what conditions equation (22) can have solutions that give rise to a proper compensator $C(s) \in \mathbb{R}_{p r}(s)^{m \times p}$. For a particular type of closed-loop denominator this problem has been studied and solved by several authors (see [6],[7],[13], [8]) and a parametrization of all possible proper denominator assigning compensators has been given (see [9], [14]). According to this approach the desired denominator is chosen to be rowcolumn reduced with particular row and column degrees in order to be able to apply degree control on the numerator and denominator of $C(s)$.

The contribution of the present paper is to provide a numerical algorithm which employes Wolovich's resultant proposed in the previous section to obtain a parametrization of all denominator assigning proper compensators. Let $X_{L}(s), Y_{L}(s)$ be a solution of (22) for a particular choice of $D_{C}(s)$ and let $k-1$ be the maximum degree of $s$ occurring amongst the elements of the matrix $\Omega(s):=\left[X_{L}(s), Y_{L}(s)\right] \in \mathbb{R}[s]^{m \times(m+p)}$. Then $\Omega(s)$ can be written

$$
\Omega(s)=\bar{\Omega}_{k} S_{k}(s)
$$

where $\bar{\Omega} \in \mathbb{R}^{m \times k(p+m)}$ and $S_{k}(s)$ as defined in (11). Then (22) can be written as

$$
\bar{\Omega}_{k} M_{e k} S_{e k}(s)=D_{C}(s)
$$

with $S_{e k}(s)$ defined if (12). Comparing the degrees of $s$ in both sides of (25) it is easily seen that $\operatorname{deg}_{c i} D_{C}(s) \leq k_{i}+k-1, i \in \mathbf{m}$ thus $D_{C}(s)$ can be written as

$$
\begin{array}{r}
D_{C}(s)=\bar{D}_{k} S_{e k}(s), \bar{D}_{k} \in \mathbb{R}^{m \times\left(\sum_{i=1}^{m} k_{i}+m k\right)} \text { and (25) becomes } \\
\bar{\Omega}_{k} M_{e k} S_{e k}(s)=\bar{D}_{k} S_{e k}(s)
\end{array}
$$

or equivalently

$$
\bar{\Omega}_{k} M_{e k}=\bar{D}_{k}
$$


since (26) must hold for every $s \in \mathbb{C}$. Thus every solution of $(22)$ can be determined from a set of numerical equations of the form (27) given the maximum degree of $\Omega(s)$ and selecting the appropriate $k$.

The following lemma can be found in [9] stated for the dual of equation (22), i.e. for a left MFD of $P(s)$. For our purposes we shall state the corresponding assumptions and the result for a right MFD of $P(s)$.

Lemma 7 ([9], Lemma 2) Consider equation (22) under the following assumptions

1. $D_{R}(s)$ is column proper with column degrees $k_{i}=\operatorname{deg}_{c i} D_{R}(s), i \in \mathbf{m}$

2. $D_{R}(s), N_{R}(s)$ are right coprime

3. $P(s)=D_{R}^{-1}(s) N_{R}(s)=N_{L}(s) D_{L}^{-1}(s)$ is strictly proper

4. $N_{L}(s), D_{L}(s)$ are left coprime

5. $D_{L}(s)$ is row proper with row degrees $\mu_{i}=\operatorname{deg}_{r i} D_{L}(s), i \in \mathbf{p}$ and define $\mu=\max _{i \in \mathbf{p}}\left\{\mu_{i}\right\}$

6. $D_{C}(s)$ is row-column reduced with $\operatorname{deg}_{c i} D_{C}(s)=\operatorname{deg}_{r i} D_{C}(s)=k_{i}+\xi_{i}$, $i \in \mathbf{m}$ where $\xi_{i}$ are integers s.t. $\xi_{i} \geq \mu-1, i \in \mathbf{m}$.

If $X_{L}(s), Y_{L}(s)$ is a solution of (22) and $C(s)=X_{L}^{-1}(s) Y(s) \in \mathbb{R}_{p r}$ (s) then $X_{L}(s)$ is row proper with row degrees $\operatorname{deg}_{r i} X_{L}(s)=\xi_{i}, i \in \mathbf{m}$.

Notice that if $X_{L}(s)^{-1} Y(s) \in \mathbb{R}_{p r}(s)^{m \times p}$ then the row degrees of $Y_{L}(s)$ cannot exceed $\xi_{i}$, i.e. $\operatorname{deg}_{r i} Y_{L}(s) \leq \xi_{i}, i \in \mathbf{m}$ [12][13], thus the maximum degree of the $i^{\text {th }}$ row of $\Omega(s)=\left[X_{L}(s), Y_{L}(s)\right]$ will be $\xi_{i}$. Denote the rows of $\Omega(s)$ by $\omega_{i}^{\top}(s) \in \mathbb{R}[s]^{1 \times(m+p)}, i \in \mathbf{m}$. Write

$$
\omega_{i}^{\top}(s)=\sum_{j=0}^{\xi_{i}} \omega_{i j}^{\top} s^{j}, \quad \omega_{i j}^{\top} \in \mathbb{R}^{1 \times(m+p)}, i \in \mathbf{m}
$$

and define the row vectors $\bar{\omega}_{i}^{\top}=\left[\omega_{i 0}^{\top}, \omega_{i 1}^{\top}, \ldots, \omega_{i \xi_{i}}^{\top}\right] \in \mathbb{R}^{1 \times(p+m)\left(\xi_{i}+1\right)}, i \in \mathbf{m}$.

Now let $d_{i}^{\top}(s), i \in \mathbf{m}$ be the rows of $D_{C}(s)$ and using assumption 6 of lemma 7 define ${\overline{d_{i}}}^{\top} \in \mathbb{R}^{1 \times m\left(\xi_{i}+1\right)+\sum_{i=1}^{m} k_{i}}, i \in \mathbf{m}$ from the relation

$$
d_{i}^{\top}(s)={\overline{d_{i}}}^{\top} S_{e\left(\xi_{i}+1\right)}(s), i \in \mathbf{m}
$$

where $S_{e\left(\xi_{i}+1\right)}$ is the $\left(m\left(\xi_{i}+1\right)+\sum_{i=1}^{m} k_{i}\right) \times m$ matrix defined in (12).

Theorem 8 Let the assumptions (1-6) of lemma 7 hold. Then every solution pair $X_{L}(s), Y_{L}(s)$ of (22) such that $C(s)=X_{L}(s)^{-1} Y_{L}(s) \in \mathbb{R}_{p r}^{m \times p}(s)$ can be obtained from the solutions of the numerical equations

$$
\bar{\omega}_{i}^{\top} M_{e\left(\xi_{i+1}\right)}={\overline{d_{i}}}^{\top}, i \in \mathbf{m}
$$


and vice versa, i.e. every solution $\bar{\omega}_{i}^{\top}$ of (30) gives rises via (28) to a $\Omega(s)=[$ $\left.X_{L}(s), Y_{L}(s)\right]$, s.t. $C(s)=X_{L}(s)^{-1} Y_{L}(s) \in \mathbb{R}_{p r}^{m \times p}(s)$.

Proof. First notice that (30) are always solvable for arbitrary ${\overline{d_{i}}}^{T}$ since $\xi_{i}+1 \geq$ $\mu$ and thus from lemma 5 in conjunction with assumptions 1-2 of lemma 7 $M_{e\left(\xi_{i}+1\right)}$ has full column rank.

If $X_{L}(s), Y_{L}(s)$ is a solution of $(22)$ and $X_{L}^{-1}(s) Y_{L}(s)$ is proper according to lemma 7 the row degrees of $\Omega(s)$ will be $\xi_{i}$ and thus we can write $\omega_{i}^{\top}(s)$ as in (28). It is easy to see that the corresponding $\bar{\omega}_{i}^{\top}$ will satisfy (30).

Conversely, if $\bar{\omega}_{i}^{\top}$ satisfy equations (30) then post-multiplying $(30)$ by $S_{e\left(\xi_{i}+1\right)}(s)$ gives $\bar{\omega}_{i}^{\top} M_{e\left(\xi_{i+1}\right)} S_{e\left(\xi_{i}+1\right)}(s)={\overline{d_{i}}}^{\top} S_{e\left(\xi_{i}+1\right)}(s), i \in \mathbf{m}$ or equivalently from $(12)$

$$
\bar{\omega}_{i}^{\top} S_{\left(\xi_{i}+1\right)}(s)\left[\begin{array}{c}
D_{R}(s) \\
N_{R}(s)
\end{array}\right]=d_{i}^{\top}(s), i \in \mathbf{m}
$$

which equivalently gives

$$
\omega_{i}^{\top}(s)\left[\begin{array}{c}
D_{R}(s) \\
N_{R}(s)
\end{array}\right]=d_{i}^{\top}(s), i \in \mathbf{m}
$$

Obviously $\Omega(s)=\left[\omega_{1}^{\top}(s), \omega_{2}^{\top}(s), \ldots, \omega_{m}^{\top}(s)\right]^{\top}$ satisfies $(22)$ and $\operatorname{deg}_{r i} \Omega(s) \leq$ $\xi_{i}, i \in \mathbf{m}$. Hence $\operatorname{deg}_{r i} X(s) \leq \xi_{i}$ and $\operatorname{deg}_{r i} Y(s) \leq \xi_{i}, i \in \mathbf{m}$.

Now let $\Lambda_{k}(s)=\operatorname{diag}\left\{s^{k_{1}}, s^{k_{2}}, \ldots, s^{k_{m}}\right\}, \Lambda_{\xi}(s)=\operatorname{diag}\left\{s^{\xi_{1}}, s^{\xi_{2}}, \ldots, s^{\xi_{m}}\right\}$ and pre and post-multiply (22) respectively by $\Lambda_{\xi}(s)^{-1}$ and $\Lambda_{k}(s)^{-1}$ to get

$$
\Lambda_{\xi}(s)^{-1} X_{L}(s) D_{R}(s) \Lambda_{k}(s)^{-1}+\Lambda_{\xi}(s)^{-1} Y_{L}(s) N_{R}(s) \Lambda_{k}(s)^{-1}=\Lambda_{\xi}(s)^{-1} D_{C}(s) \Lambda_{k}(s)^{-1}
$$

Since $D_{R}(s)$ is column proper with column degrees $k_{i}, D_{R}(s) \Lambda_{k}(s)^{-1}$ is biproper. Similarly since $D_{C}(s)$ is row-column reduced with row powers $\xi_{i}$ and column powers $k_{i}, \Lambda_{\xi}(s)^{-1} D_{C}(s) \Lambda_{k}(s)^{-1}$ is also biproper. Using the fact that $P(s)$ is strictly proper $\operatorname{deg}_{c i} N_{R}(s)<k_{i}, i \in \mathbf{m}$ thus $N_{R}(s) \Lambda_{k}(s)^{-1}$ is strictly proper. Finally, since $\operatorname{deg}_{r i} X(s) \leq \xi_{i}$ and $\operatorname{deg}_{r i} Y(s) \leq \xi_{i}, i \in \mathbf{m}, \Lambda_{\xi}(s)^{-1} X_{L}(s)$ and $\Lambda_{\xi}(s)^{-1} Y_{L}(s)$ are proper in general. Thus taking limits for $s \rightarrow \infty$ on both sides of (32) we obtain the equation

$$
X_{L}^{h r} D_{R}^{h c}=D_{C}^{h r c}
$$

where $X_{L}^{h r}$ is the highest row degree coefficient matrix of $X_{L}(s), D_{R}^{h c}$ is the highest column degree coefficient matrix of $D_{R}(s)$ and $D_{C}^{h r c}$ is the highest rowcolumn power coefficient matrix of $D_{C}(s)$. Obviously $X_{L}^{h r}$ is invertible since $D_{R}^{h c}, D_{C}^{h r c}$ are invertible. Hence, $X_{L}(s)$ is row proper with row degrees $\xi_{i}$ and since $\operatorname{deg}_{r i} Y(s) \leq \xi_{i}, i \in \mathbf{m}, X_{L}(s)^{-1} Y_{L}(s) \in \mathbb{R}_{p r}(s)$ is proper.

The above result allows us to determine the number of independent parameters in the parametrization of all proper denominator assigning compensators for a strictly proper plant, in terms the McMillan degree of the plant, the number of inputs and outputs and the particular choice of $\xi_{i}$ 's. 
Corollary 9 Let assumptions (1-6) of lemma 7 hold. Then the number of independent parameters in the parametrization of all denominator assigning proper compensators is

$$
v=m\left(p-\delta_{M} P(s)\right)+p \sum_{i=1}^{m} \xi_{i}
$$

Proof. Using the result of theorem 8 the degrees of freedom in the choice of $\omega_{i}^{T}(s)$ is essentially equal to the dimension of the left kernel of $M_{e\left(\xi_{i}+1\right)}$. Thus the total number of independent parameters will be $v=\sum_{i=1}^{m} \operatorname{dim}_{\mathbb{R}} \operatorname{ker} M_{e\left(\xi_{i}+1\right)}$. Now since $\xi_{i}+1 \geq \mu, \operatorname{dim}_{\mathbb{R}} \operatorname{ker} M_{e\left(\xi_{i}+1\right)}=\sum_{j=1}^{p}\left(\xi_{i}+1-\mu_{j}\right)$. Thus

$$
v=\sum_{i=1}^{m} \sum_{j=1}^{p}\left(\xi_{i}+1-\mu_{j}\right)=m p+p \sum_{i=1}^{m} \xi_{i}-m \sum_{j=1}^{p} \mu_{j}
$$

which using the fact that $\delta_{M} P(s)=\sum_{j=1}^{p} \mu_{j}$ gives (33).

Notice that in case we choose $\xi_{1}=\xi_{2}=\ldots=\xi_{m}:=\xi$ we don't need to solve (30) independently for each row, but we can use one resultant, namely $M_{e(\xi+1)}$ to determine all rows $\omega_{i}^{\top}(s)$. In such a case the number of independent parameters in the parametrization will be $v=m\left(p(\xi+1)-\delta_{M} P(s)\right)$.

Although theorem 8 provides a way to reduce the computation of proper compensators to the solution of a set of numerical equations of the form (30), we can go a step further and propose a method that reduces the problem to a single numerical equation. This can be done by exploiting the shift invariant form of the generalized Wolovich resultant and using Gaussian elimination.

Let $i_{1}, i_{2}, \ldots, i_{m}$ be indices such that $\xi_{i_{1}} \leq \xi_{i_{2}} \leq \ldots \leq \xi_{i_{m}}$. Let also $\xi:=$ $\xi_{i_{m}}=\max _{i \in \mathbf{m}}\left\{\xi_{i}\right\}$. In order to solve equation (30) for $i=i_{1}$ we can apply Gaussian elimination on the columns of $M_{e\left(\xi_{i_{1}}+1\right)}$ to obtain the reduced column echelon form $R_{e\left(\xi_{i_{1}}+1\right)}$. Due to the shift invariant form of the resultant, the columns of $M_{e\left(\xi_{i_{1}}+1\right)}$ appear in the first $(p+m) \xi_{i_{1}}$ rows of $M_{e\left(\xi_{i_{2}}+1\right)}$ (together with $m$ zero columns). Since $M_{e\left(\xi_{i_{1}}+1\right)}$ has full column rank, the reduced column echelon form of $M_{e\left(\xi_{i_{2}}+1\right)}$ will have the block triangular form

$$
R_{e\left(\xi_{i_{2}}+1\right)}=\left[\begin{array}{cc}
R_{e\left(\xi_{i_{1}}+1\right)} & 0 \\
Q_{11} & Q_{12}
\end{array}\right]
$$

Proceeding inductively it is easy to see that $R_{e\left(\xi_{i_{j+1}+1}\right)}$ will also have a similar block triangular form

$$
R_{e\left(\xi_{i_{j+1}}+1\right)}=\left[\begin{array}{cc}
R_{e\left(\xi_{i_{j}}+1\right)} & 0 \\
Q_{j 1} & Q_{j 2}
\end{array}\right]
$$

for $j=1,2, \ldots, m-1$. Thus reducing $M_{e(\xi+1)}$ into column echelon form, essentially provides a solution to all equations (30) since $R_{e(\xi+1)}$ consists of blocks

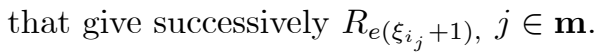


In the light of the above analysis we provide the following algorithm:

- Step 1. Obtain a right coprime MFD $N_{R}(s) \in \mathbb{R}[s]^{p \times m}, D_{R}(s) \in \mathbb{R}[s]^{m \times m}$ of $P(s)$ with $D_{R}(s)$ column proper with column degrees $\operatorname{deg}_{c i} D_{R}(s)=$ $k_{i}, i \in \mathbf{m}$.

- Step 2. Determine the minimum $k$ for which $M_{e k}$ has full column rank. Then $\mu=k$ and choose $\xi_{i} \geq \mu-1, i \in \mathbf{m}$.

- Step 3. Using (12) construct the generalized Wolovich resultant $M_{e(\xi+1)}$ where $\xi=\max _{i \in \mathbf{m}}\left\{\xi_{i}\right\}$.

- Step 4. Choose $D_{C}(s) \in \mathbb{R}[s]^{m \times m}$ to be row-column reduced with column powers $k_{i}$, and row powers $\xi_{i}$ and construct $\bar{D}_{(\xi+1)}$ by decomposing $D_{C}(s)=\bar{D}_{(\xi+1)} S_{e(\xi+1)}(s)$ as in $(26)$.

- Step 5. Construct the compound matrix $\bar{M}_{e(\xi+1)}=\left[\begin{array}{c}M_{e(\xi+1)} \\ \bar{D}_{(\xi+1)}\end{array}\right]$

- Step 6. Reduce $\bar{M}_{e(\xi+1)}$ into column echelon form to obtain $\bar{R}_{e(\xi+1)}=$ $\left[\begin{array}{c}R_{e(\xi+1)} \\ \Delta_{(\xi+1)}\end{array}\right]$

- Step 7. Compute the (general) solution for each row $\bar{\omega}_{i}^{\top}$ for $i=1,2, \ldots, m$, using the first $\left(\xi_{i}+1\right)(p+m)$ rows of $\bar{R}_{e(\xi+1)}$ and the $i^{\text {th }}$ row of $Z_{(\xi+1)}$ (discarding the last $\left(\xi-\xi_{i}\right) m$ columns on both matrices because they contain only zeroes).

- Step 8. Using (28) calculate $\omega_{i}^{\top}(s)$ of $\Omega(s)$ from $\bar{\omega}_{i}^{\top}$ for $i=1,2, \ldots, m$

Notice that the above method does not require calculation of a left coprime MFD of $P(s)$ for the parametrization of solutions as in [9] or [14] nor the computation of a $Y$-minimal particular solution as in [9]. The only information that affects the choice of the closed loop denominator is the observability index $\mu$ of $P(s)$ which can be determined using rank tests on $M_{e k}$ for successive choices of $k=1,2,3, \ldots$, since due to remark $6 \mu$ is equal to the minimum $k$ for which $M_{e k}$ has full column rank. This fact justifies the choice of the lower bound for the row powers $\xi_{i}$ of the desired closed loop denominator. In the previous section we show that $k \geq \mu$ is necessary and sufficient condition (provided that $D_{R}(s), N_{R}(s)$ are coprime and $D_{R}(s)$ is column proper) in order $M_{e k}$ to have full column rank, imposing this way the lower bound for the choice of $\xi_{i}$ 's that make equations (30) solvable for arbitrary choice of the right hand side matrix. This lower bound on the choice of $\xi_{i}$ 's has been used in the past but has not justified via some theoretic argument. With $\xi_{i}=\mu-1, i \in \mathbf{m}$, the McMillan degree of the controler $C(s)=X_{L}(s)^{-1} Y_{L}(s)$ is genericaly $\delta_{M} C(s)=m(\mu-1)$. However, there might be cases when $X_{L}(s), Y_{L}(s)$ turn out to have a left (non-unimodular) common divisor, giving rise to a $C(s)$ with McMillan degree $\delta_{M} C(s)<m(\mu-1)$. 
We should also notice that the Gaussian elimination method has been chosen here only for simplicity of presentation. The above algorithm can be applied equally well using unitary Householder's transformations to reduce $M_{e(\xi+1)}$ to a lower (block) triangular form, which performs better from a numerical point of view.

We demonstrate the above procedure via the following example (The plant and MFD's appear in the example in [14] but the desired closed loop denominator has been changed in order to illustrate the method for $\xi_{1} \neq \xi_{2}$ ).

Example 10 Let

$$
P(s)=\left[\begin{array}{ll}
\frac{s+1}{s(s-2)} & 0 \\
\frac{1}{s(s-1)} & \frac{1}{s-1}
\end{array}\right]
$$

with

$$
D_{R}(s)=\left[\begin{array}{cc}
s^{2}-2 s & 0 \\
1 & s-1
\end{array}\right], N_{R}(s)=\left[\begin{array}{cc}
s+1 & 0 \\
1 & 1
\end{array}\right]
$$

so that $k_{1}=2, k_{2}=1$ and $\delta_{M} P(s)=k_{1}+k_{2}=3$. The observability index of $P(s)$ is $\mu=2$, since it can be easily seen that $M_{e 1}$ does not have full column rank while $M_{e 2}$ does. Let the desired closed loop denominator polynomial matrix be

$$
D_{C}(s)=\operatorname{diag}\left\{s^{3}+8 s^{2}+24 s+32, s^{3}+15 s^{2}+62 s+48\right\}
$$

with $\xi_{1}=1, \xi_{2}=2, \xi=\max \left\{\xi_{i}\right\}=2$. We should expect the parametrization of all proper compensators giving rise to $D_{C}(s)$, to have $m\left(p-\delta_{M} P(s)\right)+$ $p \sum_{i=1}^{m} \xi_{i}=4$ independent parameters. Create the generalized Wolovich resultant for $k=\xi+1=3$

$$
M_{e 3}=\left[\begin{array}{ccccccccc}
0 & -2 & 1 & 0 & 0 & 0 & 0 & 0 & 0 \\
1 & 0 & 0 & 0 & 0 & -1 & 1 & 0 & 0 \\
1 & 1 & 0 & 0 & 0 & 0 & 0 & 0 & 0 \\
1 & 0 & 0 & 0 & 0 & 1 & 0 & 0 & 0 \\
0 & 0 & -2 & 1 & 0 & 0 & 0 & 0 & 0 \\
0 & 1 & 0 & 0 & 0 & 0 & -1 & 1 & 0 \\
0 & 1 & 1 & 0 & 0 & 0 & 0 & 0 & 0 \\
0 & 1 & 0 & 0 & 0 & 0 & 1 & 0 & 0 \\
0 & 0 & 0 & -2 & 1 & 0 & 0 & 0 & 0 \\
0 & 0 & 1 & 0 & 0 & 0 & 0 & -1 & 1 \\
0 & 0 & 1 & 1 & 0 & 0 & 0 & 0 & 0 \\
0 & 0 & 1 & 0 & 0 & 0 & 0 & 1 & 0
\end{array}\right] \in \mathbb{R}^{12 \times 9}
$$

Write $D_{C}(s)$ in terms of its coefficients as follows

$$
\begin{gathered}
D_{C}(s)=\bar{D}_{3} S_{e 3}(s) \\
=\left[\begin{array}{ccccccccc}
32 & 24 & 8 & 1 & 0 & 0 & 0 & 0 & 0 \\
0 & 0 & 0 & 0 & 0 & 48 & 62 & 15 & 1
\end{array}\right] S_{e 3}(s)
\end{gathered}
$$


Now define the compound matrix $\bar{M}_{e 3}=\left[\begin{array}{l}M_{e 3} \\ \bar{D}_{3}\end{array}\right]$ and apply Gaussian elimination on the columns of $\bar{M}_{e 3}$ to obtain the column echelon form which is

$$
\bar{R}_{e 3}=\left[\begin{array}{ccccccccc}
1 & 0 & 0 & 0 & 0 & 0 & 0 & 0 & 0 \\
0 & 1 & 0 & 0 & 0 & 0 & 0 & 0 & 0 \\
0 & 0 & 1 & 0 & 0 & 0 & 0 & 0 & 0 \\
0 & 0 & 0 & 1 & 0 & 0 & 0 & 0 & 0 \\
0 & 0 & 0 & 0 & 1 & 0 & 0 & 0 & 0 \\
0 & 0 & 0 & 0 & 0 & 1 & 0 & 0 & 0 \\
0 & 0 & 0 & 0 & 0 & 0 & 1 & 0 & 0 \\
-1 & 1 & -2 & 1 & 0 & 0 & 1 & 0 & 0 \\
0 & 0 & 0 & 0 & 0 & 0 & 0 & 1 & 0 \\
0 & 0 & 0 & 0 & 0 & 0 & 0 & 0 & 1 \\
1 & 0 & 0 & 0 & 1 & 0 & 2 & 0 & 0 \\
0 & 1 & -2 & 1 & 0 & 1 & 1 & 0 & 0 \\
\hline 6 & 0 & 32 & 0 & 1 & 0 & 4 & 0 & 0 \\
-63 & 78 & -204 & 126 & 0 & 16 & 62 & 0 & 1
\end{array}\right]=\left[\begin{array}{c}
R_{e 3} \\
\Delta_{3}
\end{array}\right]
$$

where $R_{e 3} \in \mathbb{R}^{12 \times 9}, \Delta_{3} \in \mathbb{R}^{2 \times 9}$. To determine $\omega_{1}^{T}(s)$ take the first $(p+m)\left(\xi_{1}+\right.$ $1)=8$ rows of $\bar{R}_{e 3}$ as well as the first row of $\Delta_{3}$ discarding the last two columns on both matrices. This corresponds to the reduced echelon form of equation (30) for $i=1$, i.e.

$$
\bar{\omega}_{1}^{\top}\left[\begin{array}{ccccccc}
1 & 0 & 0 & 0 & 0 & 0 & 0 \\
0 & 1 & 0 & 0 & 0 & 0 & 0 \\
0 & 0 & 1 & 0 & 0 & 0 & 0 \\
0 & 0 & 0 & 1 & 0 & 0 & 0 \\
0 & 0 & 0 & 0 & 1 & 0 & 0 \\
0 & 0 & 0 & 0 & 0 & 1 & 0 \\
0 & 0 & 0 & 0 & 0 & 0 & 1 \\
-1 & 1 & -2 & 1 & 0 & 0 & 1
\end{array}\right]=\left[\begin{array}{lllllll}
6 & 0 & 32 & 0 & 1 & 0 & 4
\end{array}\right]
$$

whose general solution is

$$
\bar{\omega}_{1}^{\top}=\left[\begin{array}{llllllll}
6 & 0 & 32 & 0 & 1 & 0 & 4 & 0
\end{array}\right]+\left[\begin{array}{llllllll}
1 & -1 & 2 & -1 & 0 & 0 & -1 & 1
\end{array}\right] t_{1}
$$

where $t_{1} \in \mathbb{R}$. Thus from (28)

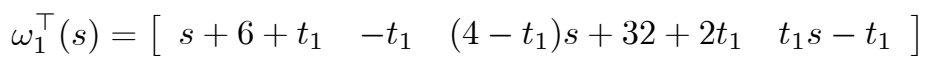

Accordingly, to determine $\omega_{2}^{T}(s)$ take the first $(p+m)\left(\xi_{2}+1\right)=12$ rows of $\bar{R}_{e 3}$ as well as the second row of $\Delta_{3}$. This corresponds to the reduced echelon 
form of equation (30) for $i=2$, i.e.

$$
\bar{\omega}_{2}^{\top}\left[\begin{array}{ccccccccc}
1 & 0 & 0 & 0 & 0 & 0 & 0 & 0 & 0 \\
0 & 1 & 0 & 0 & 0 & 0 & 0 & 0 & 0 \\
0 & 0 & 1 & 0 & 0 & 0 & 0 & 0 & 0 \\
0 & 0 & 0 & 1 & 0 & 0 & 0 & 0 & 0 \\
0 & 0 & 0 & 0 & 1 & 0 & 0 & 0 & 0 \\
0 & 0 & 0 & 0 & 0 & 1 & 0 & 0 & 0 \\
0 & 0 & 0 & 0 & 0 & 0 & 1 & 0 & 0 \\
-1 & 1 & -2 & 1 & 0 & 0 & 1 & 0 & 0 \\
0 & 0 & 0 & 0 & 0 & 0 & 0 & 1 & 0 \\
0 & 0 & 0 & 0 & 0 & 0 & 0 & 0 & 1 \\
1 & 0 & 0 & 0 & 1 & 0 & 2 & 0 & 0 \\
0 & 1 & -2 & 1 & 0 & 1 & 1 & 0 & 0
\end{array}\right]=\left[\begin{array}{llllllllll}
-63 & 78 & -204 & 126 & 0 & 16 & 62 & 0 & 1
\end{array}\right]
$$

whose general solution is

$$
\begin{aligned}
& \bar{\omega}_{2}^{\top}=\left[\begin{array}{llllllllllll}
-63 & 78 & -204 & 126 & 0 & 16 & 62 & 0 & 0 & 1 & 0 & 0
\end{array}\right]+ \\
& {\left[\begin{array}{lll}
t_{2} & t_{3} & t_{4}
\end{array}\right]\left[\begin{array}{cccccccccccc}
1 & -1 & 2 & -1 & 0 & 0 & -1 & 1 & 0 & 0 & 0 & 0 \\
-1 & 0 & 0 & 0 & -1 & 0 & -2 & 0 & 0 & 0 & 1 & 0 \\
0 & -1 & 2 & -1 & 0 & -1 & -1 & 0 & 0 & 0 & 0 & 1
\end{array}\right]}
\end{aligned}
$$

where $t_{2}, t_{3}, t_{4} \in \mathbb{R}$. Thus from (28)

$$
\begin{aligned}
\omega_{2}^{\top}(s)= & {\left[\begin{array}{ll}
-63+t_{2}-t_{3}-s t_{3} & 78-t_{2}-t_{4}+s\left(16-t_{4}\right)+s^{2} \\
& -204+2 t_{2}+2 t_{4}+s\left(62-t_{2}-2 t_{3}-t_{4}\right)+s^{2} t_{3} \quad 126-t_{2}-t_{4}+s t_{2}+s^{2} t_{4}
\end{array}\right] }
\end{aligned}
$$

Now $\Omega(s)=\left[X_{L}(s), Y_{L}(s)\right]=\left[\begin{array}{c}\omega_{1}^{\top}(s) \\ \omega_{2}^{\top}(s)\end{array}\right]$, thus the parametrization of all proper compensators is

$$
\begin{aligned}
X_{L}(s) & =\left[\begin{array}{ll}
s+6+t_{1} & -t_{1} \\
-63+t_{2}-t_{3}-s t_{3} & 78-t_{2}-t_{4}+s\left(16-t_{4}\right)+s^{2}
\end{array}\right] \\
Y_{L}(s) & =\left[\begin{array}{ll}
\left(4-t_{1}\right) s+32+2 t_{1} & t_{1} s-t_{1} \\
-204+2 t_{2}+2 t_{4}+s\left(62-t_{2}-2 t_{3}-t_{4}\right)+s^{2} t_{3} & 126-t_{2}-t_{4}+s t_{2}+s^{2} t_{4}
\end{array}\right]
\end{aligned}
$$

for free parameters $t_{1}, t_{2}, t_{3}, t_{4} \in \mathbb{R}$. Notice that the number of parameters is the expected one, i.e. 4. Obviously $X_{L}(s)$ is row proper with row degrees 1,2 while the corresponding row degrees of $Y_{L}(s)$ do not exceed 1,2 . Thus $X_{L}^{-1}(s) Y_{L}(s)$ is proper.

\section{Conclusions}

In this paper we have investigated the problem of the determination of a proper denominator assigning compensator for the class of strictly proper linear multivariable plants. Our approach focuses on the numerical computation of the 
coefficients of the polynomial matrices that describe the dynamic compensator and a parametrization of all such compensators corresponding to the one in [9] and [14] has been provided.

The suggested method utilizes a generalized version of the resultant attributed to Wolovich (see [1]) whose structural properties surprisingly have not been studied in detail. In the light of the results presented in section 3 the generalized Wolovich resultant is proved to be the ideal tool for handling polynomial matrix Diophantine equations when degree control of the solution is required. The entire procedure is reduced to the computation of a solution of a set of numerical equations and the determination of the left kernel of the generalized Wolovich resultant. Furthermore, our analysis shows that the number of independent parameters in the parametrization of all proper compensators can be calculated beforehand in terms of the row powers of the closed loop denominator and the McMillan degree of the plant.

\section{References}

[1] Wolovich, W.A. (1974), Linear Multivariable Systems. Springer Verlag. New York.

[2] Hayton G.E. (1980), The Generalized resultant matrix, International J. of Control, 32, No 4, 567-579.

[3] Bitmead R., Kung S, Anderson B., Kailath T. (1978), Greatest common divisors via generalized Sylvester and Bezout matrices, IEEE Trans. Autom. Control, Vol AC-23, No 6, pp. 1043-1047.

[4] Forney G.D. (1975), Minimal bases of rational vector spaces, with application to multivariable linear systems, SIAM J. Contr., Vol. 13, pp. 293-520.

[5] Vardulakis, A.I.G. (1991). Linear Multivariable Control - Algebraic Analysis and Synthesis Methods. John Willey \& Sons Ltd. New York.

[6] Rosenbrock, H.H. and Hayton, G.E. (1978). The general problem of pole assignment, International J. of Control, 27, 837-852.

[7] Emre, E., (1980), The polynomial equation $Q Q_{c}+R P_{c}=\Phi$ with application to dynamic feedback, SIAM J. Control and Optimization, Vol. 18, No.6, p. 611-620.

[8] Kucera V., (1970), Discrete Linear Control: The polynomial Equation Approach, Willey, Cichester, U.K.

[9] Kucera V., Zagalak P., (1999), Proper Solutions of polynomial equations, Proc. of the 14th World Congress of IFAC, London, Elservier Science, p. 357-362

[10] Antsaklis, P.J. and Michel, A.N., (1997).Linear Systems. The McGraw Hill Companies, Inc., New York. 
[11] Antsaklis P., Gao Z., (1993), Polynomial and rational matrix interpolation: theory and control applications, Int. J. Control, Vol. 58, No 2, 349-404.

[12] Kailath, T., (1980), Linear Systems. Prentice Hall. Englewood Cliffs N.J.

[13] Callier, F.M. and Desoer, C.A. (1982). Multivariable Feedback Systems.Springer Verlag. New York.

[14] Callier, F.M., (2001), Polynomial equations giving a proper feedback compensator for a strictly proper plant, Proceedings of the IFAC/IEEE Symposium on System structure and Control, Prague, Czech Republic. 